$\xi=-1$

\title{
Performance estimation of Adaptive threshold median filter design in image Denoising
}

\author{
Shruti Bhargava Choubey *, Abhishek Choubey \\ Associate Professor,Sreenidhi Institute of Science \& Technology \\ *Corresponding author E-mail: shrutibhargava@sreenidhi.edu.in
}

\begin{abstract}
Picture denoising is utilized as a part of numerous fields like PC vision, remote detecting, medicinal imaging, apply autonomy and so forth. In a significant number of these applications the presence of rash clamour in the procured pictures is a standout amongst the most widely recognized issues. The concept of this method is to provide simple but efficient method of image de-noising using filter to improve the performance and reduce the complexity of implementation. This method use the combination of average filtering and median filtering to remove the noise and produce better results with small window size $3 \times 3$. So the image details preservation is also better with small window. Mathematical results show that quality of image is better than the other filtering methods. Hardware implementation of this method is also very easy; because less number of calculations required removing the noise. Reconfigurable hardware filters may be embedded with photo acquirements provision to gain that goal. Field programmable doorway order (FPGA) is appropriate because pipelining or parallelism facts processing. What's more, though the filtering algorithm techniques huge amount over data, however such does no longer require to shops a cluster regarding intermediate data and has the consequent properties: easy of computing or reproducible, for this reason it is suitable to be applied the usage of FPGA.
\end{abstract}

Keywords: PSNR, SSIM, Median Filter, Average Filter,Noise

\section{Introduction}

In the field of Image handling, amid the transmission and obtaining, pictures are ruined by the distinctive sort of commotion. In light of the commotion, nature of picture is diminished and different highlights like edge sharpness and example acknowledgment are additionally severely influenced. There might be commotion of Gaussian kind clamor, drive compose clamor, shot commotion or salt and pepper clamor. A pivotal research is the means by which to channel clamor caused by the nature, framework and preparing of exchanges et cetera. The commotion blended with the valuable pictures or flags and brings the specialists bunches of inconveniences. In numerous exploration zones related, for example, target identifying and following, edge recognizing and picture enlistment, picture de-noising is the initial step of process. The drive clamor has for the most part two composes: settled esteem motivation commotion and arbitrary esteem motivation clamor. The settled esteem drive commotion is otherwise called salt and pepper clamor. It is for the most part reflected by pixels having least and incentive in dim scale picture. The arbitrary esteem motivation commotion is irregular in nature and it is exceptionally hard to expel this clamor. To expel the impact of clamor, we have a few procedure of picture de-noising.

Picture de-noising is an imperative pre-handling advance for some picture examination and PC vision framework. It alludes to the errand of recuperating a decent gauge of the genuine picture from a debased perception without modifying and changing helpful structure in the picture, for example, discontinuities and edges. The need a decent picture quality is progressively required with the approach of the new advances in a different zones, for example, sight and sound, therapeutic picture investigation, aviation, video frameworks and others. To be sure, the obtained picture is frequently damaged by commotion which may have a various beginnings, for example, warm vacillations; evaluate impacts and properties of correspondence channels. It influences the perceptual nature of the picture, diminishing the energy about the picture as well as the execution of the undertaking for which the picture has been proposed. The test is to plan techniques, which can specifically smooth a debased picture without changing edges, losing critical highlights and creating dependable results.But expulsion of commotion and reclamation of unique picture causes obscuring the edges of picture. Motivation clamor causes irregular high contrast spots on the picture so drive commotion lessening is vital marvels of picture preparing.

The standard median filter for the most part utilized in light of its great execution and conservation of picture points of interest. The execution of median filter likewise relies upon the span of window of channel. Littler window safeguard the points of interest however it will cause the decrease in commotion concealment. Bigger window has incredible clamour decrease ability however picture points of interest (edges, corners, scarce differences) safeguarding is restricted. With the change in the standard median filter , there were such a significant number of channels has outlined like weighted middle channel, focus weighted middle channel, versatile middle channel, rank request middle channel and numerous other enhanced channels.

Distinctive middle channels utilizes diverse arranging calculation like consolidation sort, brisk sort, pile sort to sort the components of window. A few strategies concentrated on commotion recogni- 
tion, so there are diverse procedures to discover that the pixel is uproarious or silent, with the goal that exclusive loud pixel will be supplanted by the middle esteem and quiet pixel will be unaffected. These procedures lessen the preparing time and furthermore enhance the nature of picture.

In the proposed calculation we have enhanced the system of clamor location by enhancing the limit esteem. We have utilized two edge esteems (greatest and least), so there is anything but difficult to identify the irregular esteemed drive clamor. We additionally lessen the intricacy of count on the grounds that the limit esteems and middle esteem are ascertaining all the while.

\section{Related Work}

In a viable circumstance, since the likelihood $\mathrm{p}(1.5)$ is under 1 , every one of the pixels of a computerized picture are not adulterated with the rash commotion. What's more, when the probability of debasement isn't penny percent, it is normal that the boisterous pixel be encompassed by at any rate some sound pixels. Be that as it may, this presumption isn't valid as the commotion thickness turns out to be high. Regardless, the aggregate number of defiled pixels is not as much as the aggregate number of pixels in the picture. Consequently, it isn't required to perform sifting activity on each pixel for killing the incautious commotion. Or maybe, it is computationally practical to channel just the debased pixels leaving the sound pixels unaltered. This approach decreases the obscuring impact in the re-established picture, as the greatness of sound pixels isn't influenced by separating. Fundamentally, the commotion expulsion strategy proposed in this paper constitutes two assignments: distinguishing proof of adulterated pixels and sifting activity on those tainted pixels. Along these lines the adequacy of this plan lies on the precision and power of discovery of loud pixels and effectiveness of the separating technique utilized. Numerous specialists have proposed different strategies for finding the contorted pixels and in addition separating methods. Every one of these techniques has distinctive inadequacies and thus neglects to replicate pictures near unique ones. These are overseparating twisting, obscuring impact or high computational contribution. Likewise, as the thickness of the incautious commotion is bit by bit expanded, the nature of the picture recuperated by the current strategies correspondingly corrupts. The plan proposed here, is an enhanced incautious commotion discovery plot took after by recursive middle sifting to defeat a large number of the inadequacies saw in the current strategies.

In standard middle channel, we select window of size $\mathrm{n} x \mathrm{n}$, where $\mathrm{n}$ is an odd whole number for the most part. Let the focal pixel of the window is loud, at that point sort all components of window and select the middle an incentive from the arranged components and supplant this incentive with the uproarious focal pixel. This procedure rehashed with each pixel.

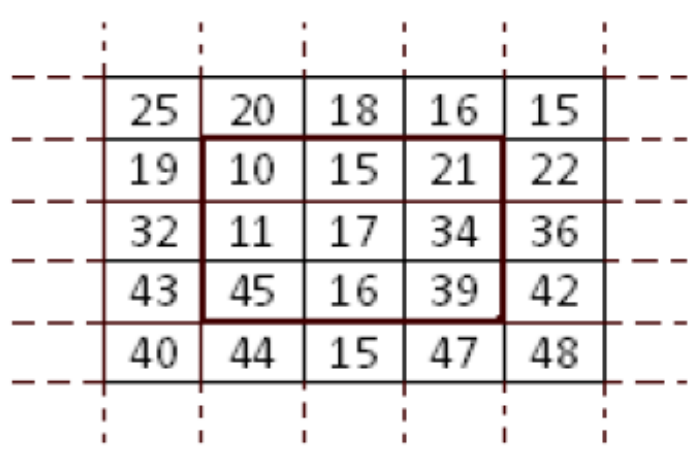

Neighborhood values:

$10,11,15,16,17,21,34,39,45$
Some of the filtering technique use the different sorting algorithm like heap sort, merge sort, quick sort to reduce the complexity of sorting. Different filters use different technique of noise detection, in which we detect that the pixel is noisy or noiseless. This has an advantage to process only noisy pixel and noiseless pixel will be remaining unchanged. This can be easily done by setting a threshold value. With the comparison of threshold value with the current pixel, we can detect that the pixel is noisy or noiseless. This technique reduces the processing time and improves the quality of image.

Content based median filter (CBMF) is an efficient method of noise de-noising. But this process contains only single value of threshold i.e. higher cut off threshold.

In the low complexity median filter, there was two threshold levels (lower cut-off threshold and upper cut-off threshold) decided for noise detection. This process selects some random values to decide the threshold levels. The detector is used to detect the noise by using these threshold values.

The other kind of median filter is weighted median filter, which filter the image according to the gray values of the pixel. It has good filtering quality of image and great application in noise removal.

The most famous median filter is adaptive median filter which has advantage to change the filtering window size according to the intensity of noise. If the pixel highly effected by noise then it adaptively increase the size of window.Some filters have high denoising quality but implementation is complex and some filter has less complex implementation but poor de-noising quality. Our main goal is to make an adjustment between the quality of output image and the implementation complexity to provide a real-time application with simple hardware. We will use the concept of pipelining to improve the de-noising operation.

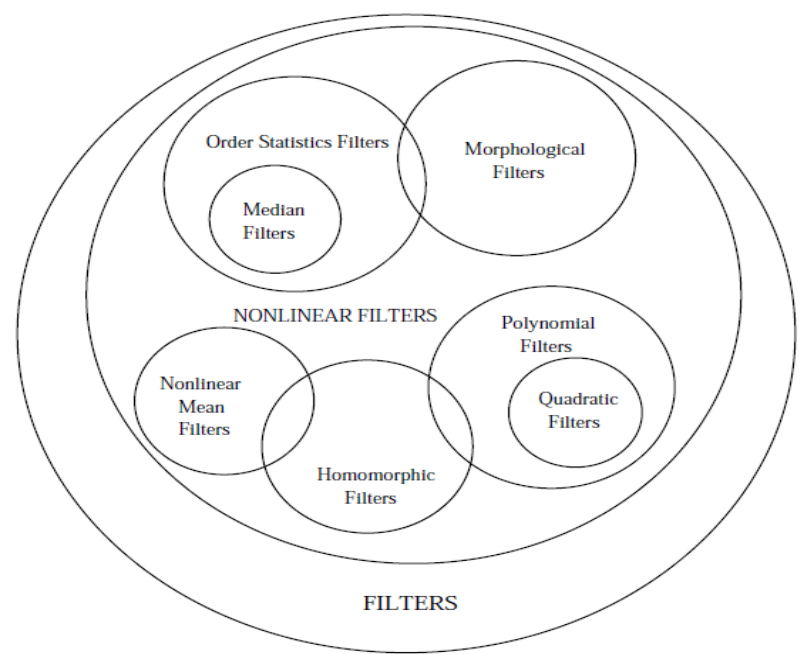

Figure.2 Types of filters for denoising

\section{Proposed Method}

As we have studied about different median filters and found that all the median filter methods use the noisy pixels in there calculation, which may effects the calculation of threshold values and median value up to some extent. So we have improved the methods by avoiding the use of noisy pixel. We use the combination of average filtering and median filtering for image de-noising. We have also used the pipelining operations which reduce the hardware requirement and complexity of circuit.

We have divided the complete process in two parts:

1) Noise detection using two threshold levels.

2) Noise removal by replacing central pixel value with median value. 
First we select the window of order ( $\mathrm{n} x \mathrm{n})$, where $\mathrm{n}$ is an odd integer generally. Now as we are examine for central pixel so we should not include it in our calculation, Because it may be noisy and effects our values and values may be not be accurate.

Now we are going to perform part one that is the detection of noise. To calculate the threshold values (minimum and maximum threshold), we use the average filtering process.

Table I: Pixels arrangements.

\begin{tabular}{|l|l|l|l|}
\hline & Column 1 & Column 2 & Column 3 \\
\hline Row 1 & $\mathrm{X}_{1}$ & $\mathrm{X}_{2}$ & $\mathrm{X}_{3}$ \\
\hline Row 1 & $\mathrm{X}_{4}$ & $\mathrm{X}_{5}$ & $\mathrm{X}_{6}$ \\
\hline Row 3 & $\mathrm{X}_{7}$ & $\mathrm{X}_{8}$ & $\mathrm{X}_{9}$ \\
\hline
\end{tabular}

We take the average of elements of first row, average of elements of last row, and save both value in an array.

$\operatorname{Avg}($ row 1$)=\left(\mathrm{X}_{1}+\mathrm{X}_{2}+\mathrm{X}_{3}\right) / 3$;

$\operatorname{Avg}($ row 2$)=\left(X_{7}+X_{8}+X_{9}\right) / 3$;

Then take the average of elements of first column, average of elements of last column and at the last taking the average of corner elements. Now save these three values in the same array.

$\operatorname{Avg}(\operatorname{clm} 1)=\left(\mathrm{X}_{1}+\mathrm{X}_{4}+\mathrm{X}_{7}\right) / 3$;

$\operatorname{Avg}($ row 1$)=\left(X_{3}+X_{6}+X_{9}\right) / 3$;

$\operatorname{Avg}(\operatorname{crn} 1)=\left(X_{1}+X_{3}+X_{7}+X_{9}\right) / 4$;

Now we have five values that do not include the noisy pixel. Now we sort these values in ascending order using sorting algorithm. The numbers of elements in the array are very less so we use the sorting algorithm possessing less complexity. After sorting the elements of array, we select three values from the array. The first element in the sorted array will be smallest one, we take the grey value of this element as minimum threshold value $\{\operatorname{Thr}(\min )\}$. Then we will select last elements of the array which is largest element of array and take it as maximum threshold value $\{\operatorname{Thr}(\max )\}$. And at last we select the middle value of array as median $\left\{\operatorname{Med}\left(\mathrm{X}_{5}\right)\right\}$. The equation of selection of these three values, minimum value, maximum value and median value are as follows:

$\operatorname{Thr}(\min )=\min \{\operatorname{Avg}($ row 1$), ., \operatorname{Avg}(\operatorname{crn} 1)\} ;$

$\operatorname{Thr}(\max )=\max \{\operatorname{Avg}($ row1 $), ., \operatorname{Avg}(\operatorname{crn} 1)\}$;

$\operatorname{Med}\left(X_{5}\right)=\operatorname{med}\{\operatorname{Avg}($ row 1$), ., \operatorname{Avg}(\operatorname{crn} 1)\}$;

So now we have three values $\operatorname{Thr}(\min ), \operatorname{Thr}(\max ), \operatorname{Med}\left(\mathrm{X}_{5}\right)$. These are minimum threshold level, maximum threshold level and median value respectively. Median value will be used for the central pixel $\mathrm{X}_{4}$. Now we will compare the value of central pixel with these two threshold values using a noise detector. If the value of central pixel is lies between these two threshold values then the pixel is noise-free and it needs not to replace with the median filter But if the value of central pixel is out of band of threshold values the pixel is considered to be noisy and we replace the value of central pixel with the median value $\operatorname{Med}\left(\mathrm{X}_{4}\right)$. Here we can see in the fig. that the average of rows and columns elements and corner elements. Here in fig.

$\mathrm{R} 1$ = average of row 1 ;

$\mathrm{R} 2$ = average of row 2 ;

$\mathrm{C} 1=$ average of column 1 ;

$\mathrm{C} 2=$ average of column 2 ;

$\mathrm{D}=$ average of corner elements;

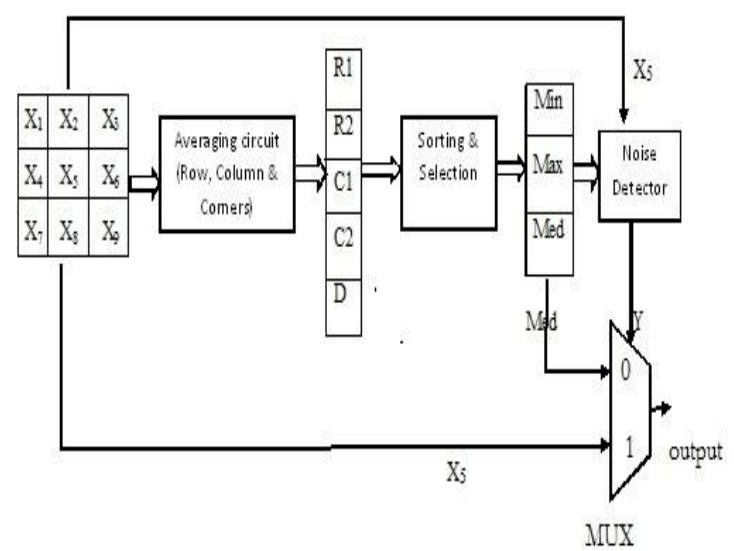

The fig shows the architecture of proposed method in which we have use averaging circuit to calculate the average of row and columns of selected window. The sorting \& selection block choose the three required values (min, max and med). Then noise detector circuit is used to perform noise detection by comparing $\mathrm{X}_{5}$ with Max and Min boundaries. The output of detector is used as selection line for noise removal circuit.

The sorting algorithm will be fastest and simple algorithm because very less number of elements are need to sort. then selecting the minimum, maximum and median values of array. The minimum and maximum values will be two corners value of sorted array. These values will be use for noise detection and median value which will be the centre value of sorted array and will be used for noise removal.

The noise detector section performs the following operation:

$\operatorname{Min}=\operatorname{Thr}(\min ), \mathbf{M a x}=\operatorname{Thr}(\max )$, Output $=\mathbf{Z}$

If $\operatorname{Thr}(\min ) \leq \mathrm{X}_{5} \leq \operatorname{Thr}(\max )$, then

$\mathrm{Y}=1$;

Output $=\mathrm{X}_{5}$,

Else $\quad \mathrm{Y}=0$;

Output $=$ Med

\section{Noise removal:-}

The noise removal block is basically a selector or we can say multiplexer. The output of noise detector will be used here as selection line for the multiplexer. If the central pixel is noise-free, the selection line will select the $\mathrm{X}_{5}$ as output. And if the central pixel is noisy then the selection line will pass the Med value to the output of multiplexer.

We will use pipelining process to calculate the average of rows and columns elements. This reduces the hardware requirements and complexity of circuit.

\section{Simulation and Results}

As the mathematical results shows that we does not include the noisy pixel in calculation so this method has great importance for the high noise density case. Because when the noise density is high, the other filter includes the noisy pixels and could not get the value as accurate as we have proposed. As the noisy pixel is avoided in calculations, this process will provide better results at high noise density and does not effects the image details unlike the other filters. With relative experimentation using MATLAB among the yardstick median filter algorithm, the median filter algorithm based on standard and the improved algorithm in the paper. 50\%, 70\%, and 90\% density impulse noise are respectively added to the original image low signal to noise ratio experiment.. Results of the enhanced algorithm in the document are shown .investigational results illustrate that the performance of the superior algorithm in the paper is better than the standard median filter algorithms(NLM,PNLM) and the fast median filter algorithm based on mean in. Especially in low signal to noise ratio circumstance, the improved algorithm has apparent advantages. 

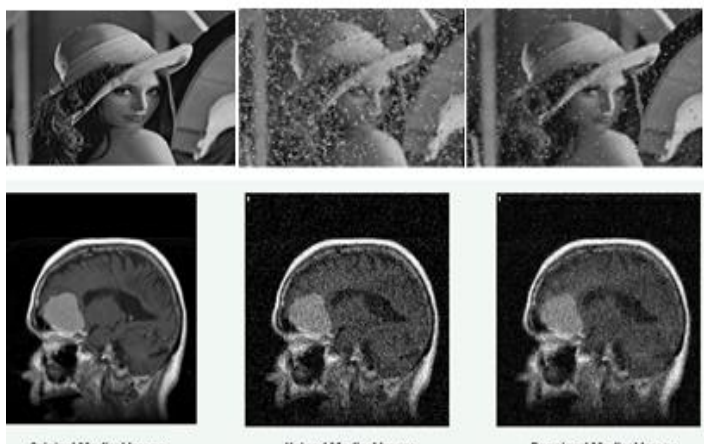

Original Medical Image
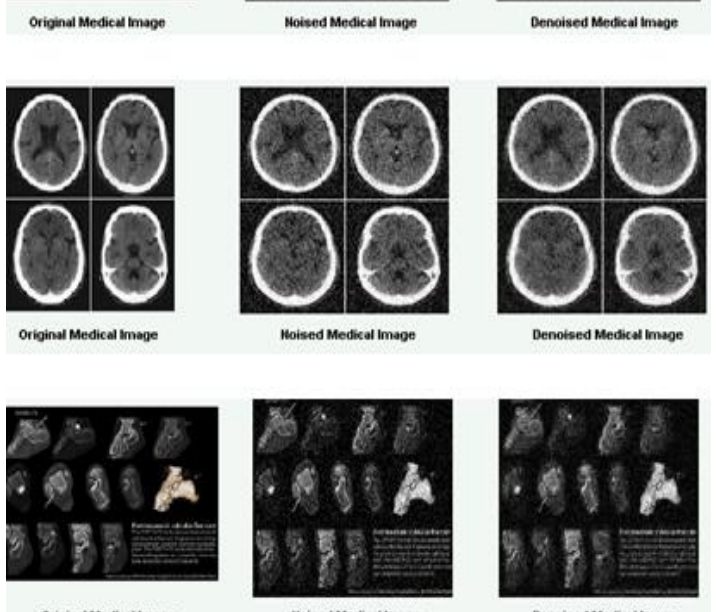

With different filtering methods [3]

(A) In terms of PSNR

\begin{tabular}{|c|l|l|l|l|l|}
\hline \multicolumn{2}{|l|}{ Image } & $\begin{array}{l}\text { ANISO- } \\
\text { TROPIC } \\
\text { FILTER } \\
\text { METHOD }\end{array}$ & $\begin{array}{l}\text { NLM } \\
\text { METH- } \\
\text { OD }\end{array}$ & $\begin{array}{l}\text { PNLM } \\
\text { METH- } \\
\text { OD }\end{array}$ & $\begin{array}{l}\text { Pro- } \\
\text { posed } \\
\text { method }\end{array}$ \\
\hline & $\begin{array}{l}\text { Noise } \\
\text { Std.de } \\
\text { v. }\end{array}$ & PSNR & PSNR & PSNR & PSNR \\
\hline Leena & 50 & 22.73 & 21.57 & 24.85 & 25.02 \\
\hline CT & 50 & 21.21 & 21.73 & 21.69 & 24.3 \\
\hline PET & 50 & 20.72 & 21.51 & 21.49 & 23.85 \\
\hline $\begin{array}{l}\text { Ultra- } \\
\text { sound }\end{array}$ & 50 & 20.16 & 22 & 23.08 & 23.89 \\
\hline Leena & 70 & 13.458 & 14.025 & 15.25 & 15.894 \\
\hline CT & 70 & 14.587 & 14.00 & 14.235 & 16.234 \\
\hline PET & 70 & 13.854 & 12.784 & 13.00 & 15.985 \\
\hline $\begin{array}{l}\text { Ultra- } \\
\text { sound }\end{array}$ & 70 & 14.254 & 14.258 & 15.874 & 16.526 \\
\hline Leena & 90 & 6.17 & 7.189 & 8.168 & 9.125 \\
\hline CT & 90 & 8.123 & 8.258 & 8.236 & 10.235 \\
\hline PET & 90 & 8.235 & 8.562 & 9.235 & 11.856 \\
\hline $\begin{array}{l}\text { Ultra- } \\
\text { sound }\end{array}$ & 90 & 9.528 & 10.425 & 10.789 & 11.845 \\
\hline
\end{tabular}

(B) In terms of SSIM

\begin{tabular}{|l|l|l|l|l|}
\hline Image & $\begin{array}{l}\text { Anisotropic } \\
\text { Filter } \\
\text { Method }\end{array}$ & $\begin{array}{l}\text { Nlm } \\
\text { Method }\end{array}$ & $\begin{array}{l}\text { (Pnlm) } \\
\text { Method }\end{array}$ & $\begin{array}{l}\text { Proposed } \\
\text { Method }\end{array}$ \\
\hline Lena & 0.6513 & 0.7993 & 0.6993 & .7125 \\
\hline CT & 0.5033 & 0.6998 & 0.6998 & .7225 \\
\hline PET & 0.5995 & 0.8952 & 0.7952 & .8258 \\
\hline Ultrasound & 0.5124 & 0.7652 & 0.6652 & .7652 \\
\hline
\end{tabular}

\section{Conclusion}

Like the other improvement in the filtering method, this proposed method is also an improvement in the standard median filtering algorithm. This method gives the median value much accurate than the other filters. So it removes the noise more efficiently than other filters. Our approach of combining the average filtering with median filtering and not including the noisy pixel give better re- sults. It is best suitable with small window size. The small window has good image details preservation. The statistical examination shows that the eminence of image is better than the standard filter and other standard median filter.

This technique has the subsequent recompense:

1) The median worth is more precise than previous filters.

2) Two doorstep used and the entry principles can adaptively change according to the noise thickness.

3) It does necessitate disconnect computation for median value and threshold standards, so it diminish the hold-up and augment the dealing out speed of filter.

4) moment perfection is the use of pipelining, which causes diminution in hardware complexity and easy in implementation.

5) Less multifaceted sorting algorithm entail because small quantity of fundamentals are need to sort.

\section{References}

[1] Kapil Kapoor1 , Dr. Abhay Sharma, De-Noising of Image Using Adaptive Thresholding Technique, International Journal of Scientific Engineering and Technology ISSN:2277-1581 Volume No.5 Issue No.3, pp: 158-160,2016.

[2] Pingjun Wei, Liang Zhang, Changzheng Ma, Tat Soon Yeo, "Fast Median Filtering Algorithm Based on FPGA," IEEE 10th International Conference on Signal Processing (ICSP), 24-28 Oct. 2010, pp. $426-429$.

[3] Balasubramanian Gopalan, A. Chilambuchelvan, S. Vijayan, and G. Gowrison, Performance Improvement Of Average Based Spatial Filters Through Multilevel Preprocessing Using Wavelets, Ieee Signal Processing Letters, Vol. 22, No. 10, October 2015.

[4] Dragana Prokin, Member, IEEE, and Milan Prokin, Member, IEEE, Fast Bit-Pipeline Rank Filter, 19th Telecommunications forum TELFOR, Serbia, Belgrade, November 22-24, 2011.

[5] Guohong LIU, Wenming GUO, "Application of improved arithmetic of median filtering de-noising, Computer Engineering and Applications," 2010, vol.46, no.10, pp.187-189

[6] Subarna Chatterjee, Ajoy Kumar Ray, Rezaul Karim, Arindam Biswas, "Architecture Design for Median Filter", Third National Conference on Computer Vision, Pattern Recognition, Image Processing and Graphics, 2011, 15-17 Dec. 2011, pp. 247 - 250.

[7] Xiaokai WANG, Feng LI, "Improved adaptive Median filtering,"Computer Engineering and Applications, 2010, vol.46, no. 3, pp. 175-176.

[8] Li Feifei, Liu Weining, "The improvement and fast implementation of median filtering algorithm with FPGA," Computer Engineering, July, vol.35, no.14, pp.175, 2009.

[9] Dragana Prokin, Member, IEEE, and Milan Prokin, Member, IEEE "Fast Bit-Pipeline Rank Filter", 19th Telecommunications forum TELFOR 2011, Serbia, Belgrade, November 22-24, 2011.

[10] WANG Chang-you, YANG Fu-ping, GONG Hui, A new kind of adaptive weighted median filter algorithm, 2010 International Conference on Computer Application and System Modeling (ICCASM 2010).

[11] Chenguang Yan and Yujing Liu, Application of Modified Adaptive Median Filter for Impulse Noise, International Conference on Intelligent Control and Information Processing - Dalian, China August 13-15, 2010.

[12] HongJun Li, ZhiMin Zhao, Image Denoising Algorithm Based on Improved Filter in Contourlet Domain, World Congress on Computer Science and Information Engineering, 2009.

[13] Shruti Bhargava, Ajay Somkuwar "Hybrid Filters based Denoising of Medical Images using Adaptive Wavelet Thresholding Algorithm" International Journal of Computer Applications ,Volume 83 - No 3, December 2013.

[14] Gerasimos Louverdis, Ioannis Andreadis and Antonios Gasteratos, "A NEW CONTENT BASED MEDIAN FILTER".

[15] Shanmugavadivu P \& Eliahim Jeevaraj P S , Laplace Equation based Adaptive Median Filter for Highly Corrupted Images, International Conference on Computer Communication and Informatics (ICCCI -2012), Jan. 10 - 12, 2012, Coimbatore, INDIA.

[16] Kh. Manglem Singh and Prabin K. Bora, Adaptive Rankordered Mean Filter for Removal of Impulse Noise from Images

[17] TANG Quan-hua, YE Jun, LEI Jin-e, Yan Zhou, A New Image Denosing Method, International Conference on Intelligent Computation Technology and Automation, 2010. 
[18] LIU Wei Computer School South China Normal University, New Method for Image Denoising while Keeping Edge Information, IEEE, 2009.

[19] S. Balasubramanian, S. Kalishwaran, R. Muthuraj, D. Ebenezer, V. Jayaraj, "An Efficient Non-linear Cascade Filtering Algorithm for Removal of High Density Salt and Pepper Noise in Image and Video sequence", International Conference on "Control, Automation, Communication and Energy Conservation" June 2009.

[20] I. Aizenberg, C. Butakoff and D. Paliy, "Impulsive noise removal using threshold boolean filtering based on the impulse detecting functions," IEEE Signal Proc. Letters, vol. 12, no. 1, pp. 63 66, 2005.

[21] Zuo-feng Zhou, Jian-zhong Cao, Hao Wang, Wei-hua Liu, Image Denoising Algorithm via Doubly Bilateral Filtering,IEEE 2009.

[22] Yueli Hu Huijie Ji, Research on Image Median Filtering Algorithm and Its FPGA Implementation, Global Congress on Intelligent Systems, 2009.

[23] Shruti Bhargava, Abhishek Choubey, Estimation of Different Thersholding Techniques for Signal Denoising, International Journal of Electronics Communication and Computer Engineering Volume 8, Issue 3, ISSN (Online):pp.153-155.

[24] Zhao Junxia, Zhen Botao and Liu Hongjun, A Median Filter FPGA with Harvard Architecture, International Conference on Information Science and Technology March 26-28, 2011 Nanjing, Jiangsu, China.

[25] Zhu Youlian, Huang Cheng, "An Improved Median Filtering Algorithm Combined with Average Filtering", IEEE, 2011 Third International Conference on Measuring Technology and Mechatronics Automation, 6-7 Jan. 2011, pp. 420-423.

[26] Shruti Bhargava and S.P.V. Subbarao, "Evaluation of Different Noise Removal Filterization Techniques in Imaging", Sreenidhi Institute of Science \& Technology,Hyderabad, International Journal of Pure and Applied Mathematics Volume 117 No. 21 2017, 605-612.

[27] Shruti bhargava, Ajay Somkuwar, Denoising Mri Images Using Wavelet Based Bivariate Shrinkage Method, CCET Journal of Science \& Engineering Education Vol. -1, Page-55-61, Year2016 